\title{
NATURALLY CONTROLLED PROCESSES AND THEORY OF RELATIVITY
}

\author{
Volodymyr Tydnjuk ${ }^{1)}$, Volodymyr Kravchenko ${ }^{2)}$, Olena Mul ${ }^{3)}$ * \\ 1) International Scientific and Educational Center of Information Technologies and Systems, 40 Academician Glushkov \\ avenue, 03680 Kiev, Ukraine, vtfly@yandex.ru \\ ${ }^{2)}$ Institute of Cybernetics named by V. Glushkov, 40 Academician Glushkov avenue, 03680 Kiev, Ukraine, \\ v_kram@i.com.ua \\ ${ }^{3)}$ Department of Mathematics, University of Aveiro, 3810-193 Aveiro, Portugal, olena@mat.ua.pt \\ * Grant from FCT (The Portuguese Foundation for Science and Technology), fellowship SFRH/BPD/14946/2004
}

\begin{abstract}
The new space-time model is proposed instead of the present model in the theories of relativity. The possible mechanism of control of some natural processes by weak space fields is demonstrated. In theoretical aspect it is raised a question about existence of an information signal along the time axis. It is shown also that existence of the maximum velocity of physical interaction propagation follows from the equations of the general relativity theory.
\end{abstract}

Keywords: General relativity theory, special relativity theory, naturally controlled processes.

\section{INTRODUCTION}

Year 2005 is a jubilee one: 100 years ago the special relativity theory was created. This theory on the strength of the non-ordinariness and its experimental verifications became so much popular now that it overstepped the limits of interests of experts in the field only.

Unfortunately, many nuances of both the special relativity theory and the general one were not investigated at all. That was caused in particular by reputation of these theories and the result was great ideological and methodical inertia of interpretation of almost all nuances of these theories and their applications. Such inertia of standardized comprehension of basic relativity theories theses causes recently an unconscious negative reaction and even attempts to return to the ether theory.

However, in both relativity theories there are some approximate things that need a modification and further development. They are: an interpretation of time as the fourth coordinate, similar to the space ones, and a full description of physical reality by continuum of four-dimensional pseudo-Euclidean geometry. As a continual space can not be described with a help of analogies of discrete substance, or ether, in the same way time is more complicated phenomenon than space. Time is most probably not a space-similar extension but a factor that "in the small" destroys and changes coherence, space structure and other topological characteristics. Time brings an irrational element in inertia of any motion, i.e. it brings an element of infinite development.

\section{STATEMENT OF PROBLEM}

A material system is considered as a controlled one if an information signal exists, which is slight in comparison with the system energy but which is able to change some system parameters significantly in comparison with its own energy. That is possible only due to a presence of a special mechanism of control and special channels of an information signal effect onto the material system. Thorough consideration of the time phenomenon presumes a hypothesis about presence of a slight information signal, connecting past, present and future events.

However, existence of such signal follows also directly from a modern form of relativity theories presentation. There, time is one of coordinates and, correspondingly, some physical interactions must be also present along the time axis, including still not known ones. Nevertheless, the next problems are still opened: is the signal along the time axis able to control a state of the material systems and physical processes at least in some cases; what are mechanisms of control and a nature of such signal?

The authors of this paper aim to answer these questions, at least partially, in the form of a noncontradictory hypothesis. It is obvious that the space-time model, which was presented in both relativity theories, needs a further development and 
modification. We show that the special relativity theory is only a consequence of the general relativity theory. Also in our next paper it will be shown that it is possible a construction of the simpler analogues of equations of the general relativity theory, out of formalism of Riemannian geometry.

\section{SOME METHODOLOGICAL PROBLEMS}

Recently a criticism of the relativity theories occurs often. However, a criticism standard should be high enough, at least not lower than the intellectual standard of these theories.

Let us consider here one of the critical papers [1], where it is proposed to consider vacuum as a twocomponent medium, or dielectric, which is exactly an universal ether. In this way it is assumed that there exist some negative and positive particles, which are not discovered yet, and they unite and form atoms of the ether, i.e. atoms of space. The author formulates averaged motion equations, which are in essence equations of deformations of the medium. The equations are written in displacements, where the displacements of two components, both for positive charges and negative ones, are present. Later equations of electrodynamics are formally derived from these equations of motion.

However, for any material medium from the equations for small mechanical displacements it is not possible to draw Coulomb's law or equations for magnetic field, as far as they are completely different energy forms. That is true even if the equations are written separately for positive and negative particles. It is impossible to build up electrodynamics without supplementary introduction into the equations of concepts of electric charges, electric field, electric currents and their interaction with magnetic field, what is absent in [1]. Also, from electrodynamics it is impossible to draw equations of heat conductivity or theory of deformations. Besides, the equations in [1] were obtained by space averaging with a grand ensemble of particles, i.e. those equations can not describe an interaction of point charges. Furthermore, with a help of very approximate formal transformations the author obtained only two Maxwell's equations, not four ones.

Here we will not go into details of the analysis of mathematical transformations and physical assumptions but we will note the essentials. About last 100 years it has not been accepted to consider any theories of ether since initially a very serious and transparent methodological error exists in any ether theory. Let us assume that a "space substance", i.e. ether, exists. Any substance is discrete, so ether consists of some particles. Then the question is: what is in spaces between the particles - ether 2, etc?

In spite of all the criticism of the Einstein theory, it is continual and describes space, but not some "substance of space". Similarly, space in the Newton theory does not consist of "ether" but "Newton continuum" is Euclidean space and "Einstein continuum" is Riemannian space.

Let us mention also some nuances, which are connected with experiments similar to Michelson ones where constancy of the light velocity and absence of any "ether wind" were demonstrated. The curvature of a light ray way in a strong gravitational field was proved theoretically and experimentally [2]. Nevertheless, a phenomenon of a possible change of the light velocity in a gravitational field has not been investigated till now. However, it is obvious from phenomenological analysis of Einstein equations that gravitating mass changes not only the curvature tensor but the metric tensor also. That must result in a change of the velocity of not only masses but probably also particles with a zero rest mass, i.e. photons. Then two rays of light, which propagate in inhomogeneous gravitational field by different ways, may pass the same distance during different time. So, in the experiments similar to the Michelson ones, inaccuracies may appear which in this way force to return to the hypothesis of "ether wind" although their real reason is very different.

Let us cite the equations of the general relativity theory in the full form $[3,4]$ :

$$
\alpha T_{i j}=R_{i j}-\frac{1}{2} R g_{i j}
$$

where $T_{i j}$ is the impulse-energy tensor, $R_{i j}$ is the Richy tensor, which is convolution, or trace of the curvature tensor, $R$ is the scalar curvature in pseudo-riemannian space, $g_{i j}$ is the metric tensor, and $\alpha$ is the numerical coefficient.

Immediately after appearance of the general theory of relativity in 1916 the attempts started to use its means for integration of all known physical fields, acting against vacuum, with the help of the "geometrical" description. First of all, that concerned gravitational and electromagnetic fields. Therefore, those attempts were the attempts to create some unified field theory [5]. Formally it means that the components of both gravitational energy and electromagnetic one should be present among the components of the impulse-energy tensor $T_{i j}$. This problem was solved in 1921 by T. Kalutsa. He showed that if to write the equations (1) not in four coordinates but in five ones, then both equations for gravitation and Maxwell's equations follow from (1).

The contemporary modernized variant of the 
Kalutsa theory, which describes the strong interaction also, is formulated in the 11-dimensional space. However, this theory is criticized a lot because of the presence of superfluous space dimensions - the real space is nevertheless a threedimensional one. Therefore, O. Klein tried to save the situation and proposed a hypothesis about compactification, or turning-ability, even at extremely short distances of superfluous dimensions.

Einstein gave too big inertia to the attempts of creation of the unified field theory but the other aspects of the general relativity theory, which are not less important, remained unexplored. First, it is an introduction of multidimensional spaces, even in a purely formal way. How can they be "situated" relatively to the real space? Then, even in multidimensional spaces if we integrate several kinds of independent forms of energy, for example, gravitational and electromagnetic ones, within the same tensor $T_{i j}$, it means that these forms are not completely independent within the limits of the problem. So, these energy forms may interact with possible transmutation as far as they are described by the same system of equations. Therefore, even if multidimensional spaces are introduced in a formal way, it is necessary to take into account that more types of physical interactions should exist in these spaces in comparison with the three-dimensional space. Apropos, that was left out of account by Ehrenfest, who tried to construct physics of multidimensional spaces.

Finally, it is unexplored at all one more important aspect of possible application of the general relativity theory. Equations (1) have a very general form. However, only two factors are considered till now as factors, changing curvature and metrics of space. They are: masses distribution energy, or energy of rest $\mu c^{2}$, generating gravitational field; and vector potentials of electromagnetic field. At the same time it is obvious that theoretically any form of energy may be presented in the impulse-energy tensor $T_{i j}$. Thus, it is shown in [6] that if we substitute a Gibbs potential into equations (1) then we have a phenomenon of a space interaction of thermochemical potentials. It denotes that in particular two spaced chemical reactions will interact through any physical medium, including vacuum. This interaction is enough weak. In [6] a quantitative estimate of the interaction constant is carried out. Besides, a hypothesis is framed that the space interaction of thermochemical potentials is exactly a basis of physical essence of information fields of living organisms.

However, by that space interactions for different forms of energy are not exhausted. If we substitute an elastic potential into (1) then we will have the phenomenon of the interaction of stressedlydeformed bodies. An analogous interaction has to exist also for different forms of mechanical motions. In essence, they are absolutely new kinds of physical interactions, which are predicted by the general relativity theory. Thus, if at least one of the abovementioned types of the space interaction is experimentally verified then the general relativity theory will at last find a wide technical application.

\section{MACROSCOPIC WAVE FUNCTION OF PROBABILITY}

Let us consider in detail a hypothesis about the information signal along the time axis. In [7] it is assumed that the macroscopic function of probability, which is similar to the wave of de Broglie, plays a role of such signal. However, the function of probability in the microcosm defines a probability of an occurrence of a particle at a determinate point of space and it is connected with the dualism "wave-particle". With regard to the macroscopic function of probability, it defines only fluctuations of some parameter under a generalized motion of macroscopic bodies.

At first sight, any motion of a macroscopic body is a strictly deterministic one. In point of fact, only not higher than the second order derivatives appear in equations of motion in the most cases.

As an example, let us consider a mechanical motion of the mass point $M_{1}$. Let us choose any scalar parameter $s_{1}$ of such motion: the coordinate of the radius vector, the projection of velocity vector or acceleration vector onto the coordinate axis, etc. Let us expand $s_{1}$ into Taylor series in the neighborhood of some moment of time $t_{0}$, cutting off after the third order derivative:

$$
\begin{aligned}
s_{1}(x, t) & \approx s_{1}\left(t_{0}\right)+s_{1}{ }^{\prime}\left(t_{0}\right)\left(t-t_{0}\right)+ \\
& +\frac{s_{1}^{\prime \prime}\left(t_{0}\right)}{2 !}\left(t-t_{0}\right)^{2}+\frac{s_{1}^{\prime \prime \prime}\left(t_{0}\right)}{3 !}\left(t-t_{0}\right)^{3},
\end{aligned}
$$

where $s_{1}\left(t_{0}\right), \quad s_{1}^{\prime}\left(t_{0}\right), \quad s_{1}^{\prime \prime}\left(t_{0}\right), \quad s_{1}^{\prime \prime \prime}\left(t_{0}\right)$ may be considered as functions of the argument $t_{0}$. Let us consider these functions as random processes, i.e. random functions of the coordinates and time, which are controlled by the normal law. For $s_{1}\left(t_{0}\right), s_{1}^{\prime}\left(t_{0}\right)$ and $s_{1}^{\prime \prime}\left(t_{0}\right)$ we can determine at each $t_{0}$ that the variance of the normal distributive law is a small quantity close to zero, i.e. these functions may be considered as very closed to the deterministic ones. 
Probability density function $p$ for the random variable $s_{1}^{\prime \prime \prime}\left(t_{0}\right)$ we will specify by the complex macroscopic function of probability:

$$
p=\psi \psi^{*}, \quad \psi=\varphi_{1}\left(t_{0}\right)+i \varphi_{2}\left(t_{0}\right),
$$

where $\varphi_{1}$ and $\varphi_{2}$ are defined by the normal distributive law. Here, $\varphi_{1}$ has an expected value, which is determined by the mean for $s_{1}^{\prime \prime \prime}\left(t_{0}-\Delta t\right)$ with a small variance. The parameters of the normal distributive law for $\varphi_{2}$ are determined in the following way.

Let us assume that the mass point $M_{2}$ exists and it is characterized by the scalar parameter $s_{2}$, which is similar to the parameter $s_{1}$. At the moment $t_{1}$ there exists enough big probability of a collision of the points $M_{1}$ and $M_{2}$, and as a result the value of the parameter $s_{1}$ may change from $s_{1}\left(t_{0}\right)$ to $s_{1}\left(t_{1}\right)$. Therefore, $\varphi_{2}$ defines the normal distributive law with the expected value $s_{1}^{\prime \prime \prime}\left(t_{1}\right)$ and the variance, which corresponds to the possibility of the collision, i.e. the function $\psi$ in the neighborhood of $t_{0}$ assumes the form:

$$
\begin{aligned}
\psi\left(t, t_{0}\right) & =\frac{1}{\sigma_{1} \sqrt{2 \pi}} \exp \left[-\frac{\left(s_{1}^{\prime \prime \prime}(t)-s_{1}^{\prime \prime \prime}\left(t_{0}\right)\right)^{2}}{2 \sigma_{1}^{2}}\right]+ \\
& +i \frac{1}{\sigma_{2} \sqrt{2 \pi}} \exp \left[-\frac{\left(s_{1}^{\prime \prime \prime}(t)-s_{1}^{\prime \prime \prime}\left(t_{1}\right)\right)^{2}}{2 \sigma_{2}^{2}}\right] .
\end{aligned}
$$

Thus, the probability density function $p=\psi \psi^{*}$ of the third order derivative at the point $t_{0}$ is a "double-humped" one, i.e. it is a sum of two normal distributive laws. Here the second "imaginary mean" is defined by an information interaction of the points $M_{1}$ and $M_{2}$ along the time axis. The function $\psi$ may be considered as a wave function:

$$
\begin{aligned}
& \psi\left(t, t_{0}, x_{0}\right)=\frac{1}{\sigma_{1} \sqrt{2 \pi}} \exp \left[-\frac{\left(s_{1}^{\prime \prime \prime}(t)-s_{1}^{\prime \prime \prime}\left(t_{0}\right)\right)^{2}}{2 \sigma_{1}^{2}}\right] \times \\
& \times \sin \left(k_{1} x_{0}-\omega_{1} t_{0}\right)+i \frac{1}{\sigma_{2} \sqrt{2 \pi}} \times \\
& \times \exp \left[-\frac{\left(s_{1}^{\prime \prime \prime}(t)-s_{1}^{\prime \prime \prime}\left(t_{1}\right)\right)^{2}}{2 \sigma_{2}^{2}}\right] \sin \left(k_{2} x_{0}-\omega^{\prime} t_{0}\right) .
\end{aligned}
$$

Besides, the higher and lower derivatives in the formula (2) are connected by the relationship:

$$
\frac{\partial s_{1}^{(n-1)}}{\partial t}\left(t_{0}+\Delta t\right)=\frac{\partial s_{1}^{(n-1)}}{\partial t}\left(t_{0}\right)+\frac{\partial s_{1}^{(n)}}{\partial t}\left(t^{\prime}\right) \Delta t .
$$

Then, considering (2), at some point between $t_{0}$ and $t_{1}$ we can expect an appearance of the second "hump" in the probability density function of the parameter $s_{1}$ directly. Therefore, taking into account (5) we can consider that the mass point $M_{1}$ has two natural frequencies $\omega_{1}$ and $\omega^{\prime}$, which characterize random fluctuations of the parameter $s_{1}$ around the deterministic function, that defines its values at time.

Thus, the fluctuations of the parameter $s_{1}$ may be given as a sum of two normal distributions, where the second normal law describes a possible interaction along the time axis. Let us write the fluctuations equation for the "second mean" of the fluctuations $q_{1}$ of the parameter $s_{1}$ for the mass point $M_{1}$ [8]. Here we should take into account that the points $M_{1}$ and $M_{2}$ have also a space interaction in view of the equation (1). The character of this interaction was in general described in the previous paragraph. We assume that the space interaction has a wave character also and it is characterized by some set of harmonics. Each such harmonic $\omega$ of the space action of the point $M_{2}$ onto the point $M_{1}$, relative to fluctuations of the parameter $s_{1}$, has disturbing force character. Thus, the fluctuation equation for each harmonic $\omega$ has the next form:

$$
q_{1}^{\prime \prime}+k^{2} q_{1}=h \sin (\omega t) .
$$

Above we defined in (5) that also the information interaction existed between the points $M_{1}$ and $M_{2}$ along the time axis with the frequency $\omega^{\prime}$, which resulted in the fluctuations of both the parameter $s_{1}$ of the point $M_{1}$ and the parameter $s_{2}$ of the point $M_{2}$ with the same frequency. Therefore, the harmonic $\omega^{\prime}$ has to be present among the harmonics $\omega$ of the space action of the point $M_{2}$ onto $M_{1}$ and the equation (7) may be written as follows:

$$
q_{1}^{\prime \prime}+k^{2} q_{1}=h \sin \left(\omega^{\prime} t\right) .
$$

The frequency $\omega^{\prime}$ is also a natural frequency of fluctuations for the parameter $q_{1}$ of the point $M_{1}$. Thus, a resonance condition takes place when the 
frequency of the disturbing force (8) coincides with the natural frequency and in this case the amplitude of fluctuations $q_{1}$ has to increase considerably [8].

Therefore, in the presence of both space interaction between the points $M_{1}$ and $M_{2}$ and the information interaction along the time axis, the parameter of motion $s_{1}$ of the point $M_{1}$ is a controlled one. At the point $t_{0}$ this parameter was characterized by the large probability for the mean $s_{1}\left(t_{0}\right)$, but at the point $t_{1}$ there exists the whole set of different possibilities for $s_{1}$, depending on the control: a) $s_{1}\left(t_{1}\right)$ is defined by almost the same deterministic function (2) that was at the point $t_{0} ; \mathrm{b}$ ) $s_{1}\left(t_{1}\right)$ is characterized by the small fluctuations of the function $(2)$; c) $s_{1}\left(t_{1}\right)$ is characterized by the large fluctuations; d) a collision of the points $M_{1}$ and $M_{2}$ takes place at the moment $t_{1}$, and $s_{1}\left(t_{1}\right)$ is a function, which is completely different from (2).

Let us write the equation of control for $s_{1}$ :

$$
\frac{d s_{1}}{d t}=A(t) s_{1}+B(t) u+C(t),
$$

where $A(t), B(t)$ and $C(t)$ are some known functions.

Here the control $u$ in the simplest case, which was analyzed, depends on the expected value of one of the normal distribution laws for the third-order derivative of $s_{1}$, which defines the interaction along the time axis (function $\varphi_{2}$ in (3)). Also the control $u$ depends on the amplitude of the harmonic $\omega^{\prime}$ of the space action of the point $M_{2}$ onto $M_{1}$. We need to note that supplementary conditions of resonance and estimation of the amplitudes of the second maximum in the sum of two normal distributions for $S_{1}$ are the subjects of more complicated analysis and they are not investigated in this paper. Thus, the information control along the time axis may be realized by means of the higher derivatives and the resonance of information signals - both the signal along the time axis and the space one.

Parameters of chemical reactions and thermal processes have the largest fluctuations. In [7] references are given to the experiments, which justify a presence of "double-humped" distributions under chemical reactions and biological processes.

In natural processes the simpler model of control may be used also, where the signal along the time axis is not considered. The fractal phenomenons may often appear in such models. For instance, a controlled function may not have continuous derivatives at all, and so "inertia of determinacy" is missing in it. According to the equation (1), any energy process generates a space field, which has a wave character often. This field may influence on the other similar physicochemical process, even with the other energy form, but probably a fractal similarity plays a role here. The signal of such space field is very weak and has an information nature. However, it may resonate with normal modes of the system, on which it influences, and in such cases it becomes a control signal. Then space, or vacuum, becomes an universal transmitter of different interactions between different forms of energy.

For instance, the magnetic field of the Earth is weak enough to generate, under self-disturbances, electric currents in living organisms, which are able to influence at organism vital functions. Nevertheless, magnetic storms influence on physiological systems. That mechanism is not completely cleared up but it can be easily explained by the presence of a space interaction, which is described in the general relativity theory. In fact, any chemical reaction of any ferment system of organism has its own set of fluctuations, including fluctuations for the rotational component of Gibbs potential. If the same frequencies appear under disturbance of the magnetic field of the Earth, then the resonance takes place in such chemical reaction by means of action of the space field on it.

Similarly, according to the principle of energy transfer and fractal similarity of processes, weak disturbances of the gravitational field of the Earth can influence on displacements of air and water masses, i.e. on the weather, as well as directly on chemical reactions and vital functions of organisms.

It is usually accepted that the bodies of the solar system, except for the Moon and probably for the Jupiter, almost do not influence on different earth processes because of weak strength of their gravitational fields at such long distances. This point of view leaves out of account the phenomenon of possible resonance of a weak information signal in material systems, on which it influences. Moreover, according to the proposed model of space interactions, any physicochemical processes at the planets of the solar system generate space information signals, which are also able to influence on different processes at the Earth.

Naturally, the existence of the weak information interaction along the time axis, exciting fluctuations of measurable parameters, is more problematical than space interactions for any form of energy, which follow directly from the equations of the general relativity theory.

Let us consider a respective phenomenological model now. Aristotle gave his very deep view about 
time as follows: "Everything in the universe consists of the whole and the parts. It is impossible to say that only about time because we always see only its part, which is the current moment. Where is that place, from which it is possible to see simultaneously both yesterday and tomorrow?"

Time is a dialectical combination of two opposite tendencies - rational and irrational ones. If some process is repeated in a material system, then it will have some new elements at least in the small: slightly different values of parameters, different fluctuations of these parameters, different interaction during the process with other material systems, etc. Simultaneously, a rational causal-investigatory relationship is inherent for the phenomenon of time, when events follow logically from their causes and are mainly predictable.

If we fix some moments of time $t_{1}$ and $t_{2}$ in a real physical world, there will not be absolutely any known physical interaction between them. Sections of the three-dimensional space at $t_{1}$ and $t_{2}$ are not related at all, at least continually. Topology of the interval between these sections may be most probably described with the help of a set with the bigger cardinality than the cardinality of the continuum. That "non-continual" interval is exactly a source of random, unpredictable and irrational in the phenomenon of time.

The presence of any hypothetical information signal between $t_{1}$ and $t_{2}$, even in the form of the macroscopic complex wave function of probability, assumes a continual relationship between $t_{1}$ and $t_{2}$. It is because $\varphi_{1}$ and $\varphi_{2}$ from the wave function (3) assume a four-dimensional "coupling" at least, as well as an interaction of three-dimensional sections, respectively described by $\varphi_{1}$ and $\varphi_{2}$. As a result, the change of events probabilities in the interval between $t_{1}$ and $t_{2}$ is assumed.

The way out is to assume, at least formally, an existence of a virtual parallel space, where the events of the physical world are mapped into, but where already a continual relationship exists between $t_{1}$ and $t_{2}$. The periodical virtual short-term interaction with the physical world generates both stable causal-investigatory relationships and a probabilistic interaction between sections at $t_{1}$ and $t_{2}$. Such model combines in the phenomenon of time simultaneously both a continual interpretation and a non-continual one.

The theories, which are similar to the Kalutsa theory, also claim at least a formal existence of multidimensional spaces. If we assume a simple "immersion" of the real three-dimensional space into the multidimensional ones, then numerous effects of interaction should be generated. However, they do not take place. Thus, a solution in this case is also an existence of the parallel multidimensional spaces.

In quantum mechanics the problem of creation of an adequate phenomenological model is very urgent. The wave probability function in the Schrodinger formulation is defined in the real space but an explanation is absent why it is complex but not real. In fact, the complex form of the wave function assumes an interaction of two three-dimensional sections, which correspond to the real and imaginary parts of this function, and such interaction is possible at the minimum in a four-dimensional space. The wave function in the Heisenberg formulation of quantum mechanics is from the outset defined in a multidimensional space whereas a real space is three-dimensional. Nevertheless, an application of that mathematics body gives correct results. Naturally, the lack of the theoretical basis and phenomenological model should be eliminated as soon as possible. In this case the creation even of a very approximate or erroneous model is better than its absolute absence.

The proposed phenomenological model allows in a completely new way to interpret the presence of the fourth continual axis, the axis of time, in the both relativity theories. This imaginary axis in the model interpretation does not belong to the real physical world, it is virtual as a result of a virtual interaction with a conjectural parallel multi-dimensionality.

The introduction of multidimensional parallel spaces and their virtual interaction is only a mathematical abstraction, without claims to a conformity with a physical reality. However, such phenomenological model gives the possibility of further development of the conception "space-time", which remains the same like it was 100 years ago.

\section{CORRELATION OF GENERAL AND SPECIAL THEORIES OF RELATIVITY}

Let us consider the equation (1) in the space coordinates, when $i, j=1,2,3$. Let us assume that the tensor $T_{i j}$ is given by an uniform and rectilinear flow of masses of an equal density. Without restriction of generality, let us assume that the motion occurs along the axis $x_{1}$. Then only one of the projections of the velocity is nonzero, i.e. $v_{1}=v=$ const $\neq 0$, and the density of masses is given by $\mu=$ const. It is natural to assume that the curvature tensor is equal to zero in this case. Therefore, from the equation (1) we obtain:

$$
\beta T_{11}=-g_{11}, \quad \beta=2 \alpha / R
$$


As a rule, for example, in the mechanical motion case, the structure of the impulse-energy tensor is given as a product of the velocity projections $v_{i} v_{j}$. In our case it is natural to assume the more generalized variant. As the second power of the length unit is present in $g_{11}$, the kinetic energy is also dependent on the second power of the velocity. So we may consider $T_{11}$ as some unknown function of the second power of the velocity $v_{1}^{2}=v^{2}$.

Let us expand $T_{11}$ into Taylor series, cutting off after the linear term:

$$
T_{11}=f\left(v^{2}\right) \approx a^{\prime}+b^{\prime} v^{2},
$$

and then

$$
g_{11}=-\beta a^{\prime}-\beta b^{\prime} v^{2}
$$

Now we pass from the coefficients $a^{\prime}, b^{\prime}$ to the non-negative coefficients $a, b$ : if, for example, $a^{\prime}<0$, then $a=-a^{\prime}$. In this case, as far as the metric tensor is a scalar product of the respective basis vectors of the curvilinear coordinate system and it can not be negative, there exist two possibilities for its determination, depending on values of the terms from the right-hand part of (12):

$$
g_{11}=\left\{\beta a-\beta b v^{2}, \quad \beta b v^{2} \mp \beta a\right\} .
$$

In the first case of (13), length shortening occurs under the motion with the constant velocity $v$. The second case corresponds to lengthening under velocity increasing. Shortening of the length under velocity increasing accords as it were additional "braking" by vacuum. So, in the second case the space should cause an additional acceleration of a material body under velocity increasing. That means that the energy does not dissipate and it comes "from nowhere". However, that is unreal so we suppose:

$$
g_{11}=\beta a-\beta b v^{2} .
$$

When the mass flow is absent, the space becomes Euclidean and $g_{11}$ is the second power of the basis vector $n$ of an orthonormal coordinate system. If the mass flow moves with some velocity, the orthonormal coordinate system becomes a curvilinear one, and the vector $n$ changes the length to $n^{\prime}$ :

$$
g_{11}=n^{\prime^{2}}=\left(\frac{l^{\prime}}{l_{0}}\right)^{2}=\beta a-\beta b v^{2},
$$

where $l_{0}$ is an arbitrary length at $v=0$, and $l^{\prime}$ is the same length at $v>0$.

At $v=0$ we have $g_{11}=n^{2}=1$, therefore from (15) it follows that $\beta a=1$ and

$$
n^{\prime}=\frac{l^{\prime}}{l_{0}}=\sqrt{1-\beta b v^{2}} .
$$

Now, assuming that $1 / \beta b=c^{2}$, we can write (16) in the next form:

$$
n^{\prime}=\frac{l^{\prime}}{l_{0}}=\sqrt{1-\frac{v^{2}}{c^{2}}} .
$$

Shortening of the length of the basis vector to $n^{\prime}$ does not assume its negative and, what is more, imaginary values. Thus, it follows from (17) the existence of the maximum velocity of interaction propagation $c$, and it is natural to assume this velocity equal to the light velocity. Also, the formula (17) directly gives Lorentz shortening of the length:

$$
l^{\prime}=l_{0} \sqrt{1-\frac{v^{2}}{c^{2}}} .
$$

From (16)-(17) it is obvious that the function of the dependence of the impulse-energy tensor on the velocity in (11) can not differ even by a constant. Therefore, from the existence of the maximum velocity of interaction propagation, which is equal for all the inertial coordinate systems, it follows that so-called interval $s$ between two events in different inertial coordinate systems is constant, or invariant:

$$
\begin{aligned}
s^{2} & =c^{2}(\Delta t)^{2}-(\Delta x)^{2}-(\Delta y)^{2}-(\Delta z)^{2}= \\
& =c^{2}\left(\Delta t^{\prime}\right)^{2}-\left(\Delta x^{\prime}\right)^{2}-\left(\Delta y^{\prime}\right)^{2}-\left(\Delta z^{\prime}\right)^{2} .
\end{aligned}
$$

As it is well known, the Lorentz transformations follow from (19) and they are axioms of the special relativity theory together with the postulate of constancy of the light velocity as a maximum velocity [9]. Therefore, the special relativity theory is not independent but it is just a consequence of the general relativity theory. The introduction of the fourth imaginary axis ict and pseudo-Euclidean geometry in view of the constancy of the interval $s$ is only an artificial mathematical technique and it is possible to omit it both in the special relativity theory and in the general one. 


\section{DISCUSSION OF THE RESULTS AND CONCLUSIONS}

If we consider the space as a Riemannian but not a Euclidean, then a change of the dependence of its basic geometrical characteristics, such as the metric tensor and the curvature tensor, on the coordinates and time is in identical with an appearance of some force field. That field is generated by energy of the material medium, or substance, which is distributed in the space in some way. Till now it has not been taken into account that, according to the concepts and equations of the general relativity theory, any form of energy generates such a specific field.

The modification of the model "space-time" of the relativity theory based on the four-dimensional pseudo-Euclidean geometry is stipulated by the next. Approximation of the time phenomenon only by a complementary space extension is very inaccurate. In physics, the principle of variations for parameters of a generalized motion is widely used. According to the principle, between points $t_{1}$ and $t_{2}$ there exists at least a continual set of possible trajectories for the motion. However, some restrictions permit to choose only one substantively possible trajectory from all those. The principle of variations reflects a basic property of the time phenomenon, which is the existence of a great many of different possibilities of development with time. A three-dimensional space is here associated with each point of each trajectory between $t_{1}$ and $t_{2}$. In some way such space is similar to the real one, corresponding to the moment $t_{1}$.

Thus, integrating all the trajectories, we have a set, which may be approximated to some extent by a set of all real space subsets that include $t_{1}$ and $t_{2}$. Such set, as it is known, has a bigger cardinality than the continuum cardinality. Thus, a construction of a non-continual part of the proposed "space-time" model instead of the four-dimensional pseudoEuclidean one is reasonable and justified by the existence of the variation principle. The second part of the model, based on the existence of virtual parallel spaces, implies a presence of a selection mechanism and implementation of a real trajectory of changes with time of physical processes from the whole non-continual set of trajectories-spaces.

Finally, it is very important that the existence of the maximum velocity of physical interaction propagation was considered before as one of the axioms of the special relativity theory. As it was shown, the existence of such velocity follows from the characteristics of the interaction of material medium energy with space as well as from the equations of the general relativity theory.
The phenomenon of influence of many information factors on water crystallization, crystal shapes, etc., was discovered by Masaru Emoto [10]. That is an indirect experimental verification of existence of a thermochemical potential field, since existing physical theories can not explain the presence of this phenomenon.

The main conclusion is that a natural control of the processes is possible by means of a weak information signal of any nature. The mechanism of such control is effected with the help of the resonance phenomenon.

Creation of detectors of a thermochemical potential field will afford more opportunities for study of spectra of absorption and emission in a nonelectromagnetic field. That is of great importance for applications in technological areas, which are based on thermal and chemical processes, as well as for the deeper study of different living organism fields and their correlation.

\section{REFERENCES}

[1] L. Khoroshun. System model of two-continuum mechanics of dielectrics as a basis of electrodynamics and theory of universal ether [in Russian], Systemni doslidgennya ta informatsijni tekhnologii 2 (2003). pp. 108-123.

[2] E. Schmutzer. Theory of relativity. Contemporary conception [in Russian]. Nauka. Moscow, 1991. p. 220.

[3] P. Rashevskij. Riemannian Geometry and Tenzor Analysis [in Russian]. Nauka. Moscow, 1967. p. 554.

[4] L. Landau. E. Lifvsic. Theory of field [in Russian]. Nauka. Moscow, 1967. p. 458.

[5] B. Parker. Albert Einstein's vision. Prometheus Books. Amherst. NY. Dordrecht, 2004. p. 286.

[6] V. Tydnjuk. F. Gorin. V. Kravchenko. On the problem of an information model in the theory of living organisms fields [in Russian], Problemy Upravlen. Inform. 2 (2003). pp. 146-152.

[7] V. Tydnjuk. V. Kravchenko. O. Mul. M. Shut. Information interaction of thermochemical potentials in living organisms and general theory of relativity, Computing 3 (2) (2004). pp. 91-98.

[8] I. Babakov. Theory of oscillations [in Russian]. Nauka. Moscow, 1965. p. 559.

[9] B. Medved. Principles of theoretical physics [in Russian]. Nauka. Moscow, 1977. p. 496.

[10]M. Emoto, D. A. Thayne (Translator). The Hidden Messages in Water. Beyond Words Pub Co. Hillsboro, 2004. p. 159. 


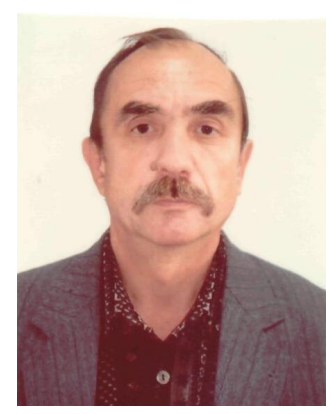

Tydnjuk

Volodymyr

Zotovych is a scientific officer of UNESCO International Research Center of Information Technologies and Systems. He graduated from the mechanico-mathematical department of Kyiv State University named by $T$. G. Shevchenko. He worked in different institutions of Academy of Sciences of Ukraine such as Institute of Mechanics, Institute of Cybernetics named by V. Glushkov, UNESCO International Research Center of Information Technologies and Systems. He is an inventor, the author of the new technique of electro-acupuncture diagnostics. The field of his scientific investigations is mathematical modeling in biology, physiology and medicine; non-ordinary applications of general theory of relativity, in particular for description of fields of living organisms; artificial intelligence.

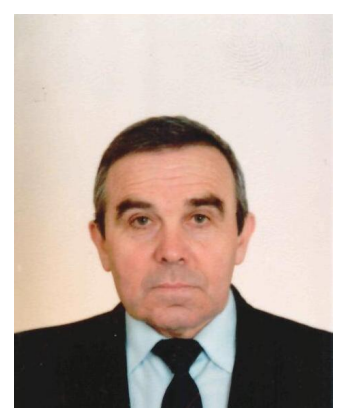

Kravchenko Volodymyr Pavlovych is Ph.D. in
Physics and Mathematics, senior scientific officer, scientific secretary of Engineering and Innovation Committee of Aerospace Association of Ukraine. He graduated from the mechanico-mathematical department of Kyiv State University named by $T$. G. Shevchenko. He worked in such institutions of Academy of Sciences of Ukraine as Institute of Mechanics, Institute of Hydromechanics, Institute of Geotechnic Mechanics, Institute of Cybernetics named by V. Glushkov. He is the author of 6 monographs and more than 150 scientific papers.

Mul Olena Vladlenivna is Ph.D. in Physics and Mathematics. The current position is a 3-year Postdoctoral fellowship at the Department Mathematics, University of Aveiro, Portugal, granted by FCT (The Portuguese Foundation for Science and

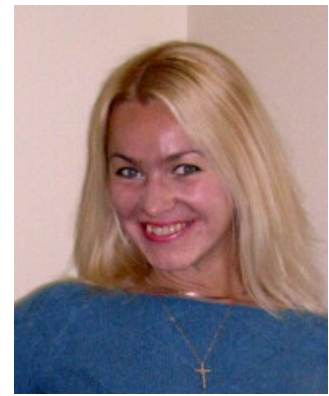

Technology). She graduated from State University "Lvivska Politechnika" by the applied mathematics specialty. She defended Ph.D. thesis "Analysis of Self-Oscillation Processes in the Complex Continuous and Discrete Systems" in Kyiv, at Academic Council of Space Research Institute of NASU and NSAU. She worked as an associate professor in the Institute of Computer Information Technologies of Ternopil Academy of National Economy and in Ternopil State Technical University. She was repeatedly awarded by grants from NATO, UNESCO, CIME, IUPAP, C.I.R.M. and others for participation in international scientific conferences and seminars. She is the author of more than 30 scientific papers in the field of analysis and control of different complex dynamical systems as well as devoted to some problems of mathematical physics and mathematical modeling. 